\title{
Analysis of Coding Techniques in WiMAX
}

\author{
Prabhakar Telagarapu \\ Dept.of.ECE \\ GMR Institute of Technology \\ Rajam, AP, India
}

\author{
G.B.S.R.Naidu \\ Dept.of.ECE \\ GMR Institute of Technology \\ Rajam, AP, India
}

\author{
K.Chiranjeevi \\ Dept.of.ECE \\ GMR Institute of Technology \\ Rajam, AP, India
}

\begin{abstract}
Coding techniques is used for providing reliable information through the transmission channel to the user. In coding techniques the number of symbols in the source encoded message is increased in a controlled manner in order to facilitate two basic objectives at the receiver one is Error detection and other is Error correction. It is used to reduce the level of noise and interferences in electronic medium. The amount of error detection and correction required and its effectiveness depends on the signal to noise ratio (SNR). In digital communication, coding techniques is a broadly used term mostly referring to the forward error correction code. The advantage of forward error correction is that a back-channel is not required, or that retransmission of data can often be avoided, at the cost of higher bandwidth requirements on average. In this paper is useful for performance evaluation of physical layer of WIMAX by using Reed-Solomon coding and convolution coding scheme, cyclic prefix and interleaving for different modulation technique with respect to bit-error rate and SNR ratio.
\end{abstract}

\section{Keywords}

Error detection, Error correction code, WIMAX cyclic prefix, $\mathrm{SN}$, Reed-Solomon coding

\section{INTRODUCTION}

The main objective of this paper is to transmit the data in WIMAX with low bit error rate in the noisy environment for that we using Forward Error Correction method which is Reed Solomon coding and Convolution coding .this method is useful to reduce the bit error rate(BER) and increase the efficiency. Reed Solomon coding and Convolution coding are the two powerful error correction and detection methods to reduce the noise. In order to increase the performance of the coding technique we are using Cyclic Prefix and interleaving techniques. So in this paper is useful for analysis of physical layer of WIMAX with different modulation techniques like BPSK, QPSK, QAM and comparison of QPSK modulation with and without Forward Error Correction methods. Broadband Wireless Access (BWA) has emerged as a promising solution for last mile access technology to provide high speed internet access in the residential as well as small and medium sized enterprise sectors. At this moment, cable and digital subscriber line (DSL) technologies are providing broadband service in this sectors. But the practical difficulties in deployment have prevented them from reaching many potential broadband internet customers. Many areas throughout the world currently are not under broadband access facilities. Even many urban and suburban locations may not be served by DSL connectivity as it can only reach about three miles from the central office switch. On the other side many older cable networks do not have return channel which will prevent to offer internet access and many commercial areas are often not covered by cable network. But with BWA this difficulties can be overcome. Because of its wireless nature, it can be faster to deploy, easier to scale and more flexible, thereby giving it the potential to serve customers not served or not satisfied by their wired broadband alternatives. IEEE 802.16 standard for BWA and its associated industry consortium, Worldwide Interoperability for Microwave Access (WIMAX) forum promise to offer high data rate over large areas to a large number of users where broadband is unavailable. This is the first industry wide standard that can be used for fixed wireless access with substantially higher bandwidth than most cellular networks. Wireless broadband systems have been in use for many years, but the development of this standard enables economy of scale that can bring down the cost of equipment, ensure interoperability, and reduce investment risk for operators. The first version of the IEEE 802.16 standard operates in the $10-66 \mathrm{GHz}$ frequency band and requires line of sight (LOS) towers. Later the standard extended its operation through different PHY specification to $211 \mathrm{GHz}$ frequency band enabling non line of sight (NLOS) connections, which require techniques that efficiently mitigate the impairment off adding and multipath. Taking the advantage of OFDM technique the PHY is able to provide robust broadband service in hostile wireless channel. The OFDM based physical layer of the IEEE 802.16 standard has been standardized in close cooperation with the European Telecommunications Standards Institute (ETSI) High Performance Metropolitan Area Network (HIPERMAN).Thus, the HIPERMAN Standard and the OFDM based physical layer of IEEE 802.16 are nearly identical. Both OFDM based physical layers shall comply with each other and a global OFDM system should emerge. The WIMAX forum certified products for BWA comply with the both standards.

\section{SIMULATION MODEL}

\subsection{Physical layer setup}

The structure of the baseband part of the implemented transmitter and receiver is shown in Figure 1. This structure corresponds to the physical layer of the IEEE 802.162004 Wireless MAN OFDM air interface. In this setup, we have just implemented the mandatory features of the specification, while leaving the implementation of optional features for future work. Channel coding part is composed of three steps randomization, Forward Error Correction (FEC) and interleaving. FEC is done in two phases through the outer Reed-Solomon (RS) and inner Convolutional Code (CC). The complementary operations are applied in the reverse order at channel decoding in the receiver end. The complete channel encoding setup is shown in Figure 2 while corresponding decoding setup is shown in Figure 3. 
Through the rest of the sections, the individual block of the setup will be discussed with implementation technique

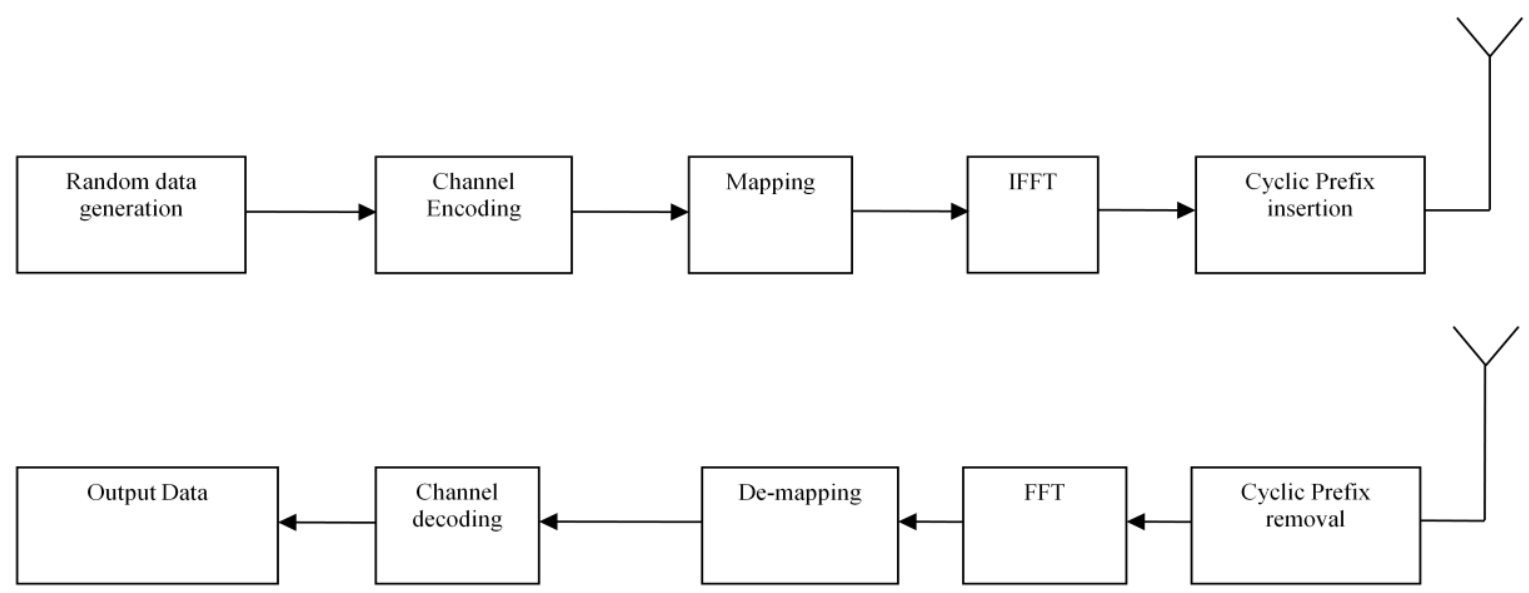

Fig 1: Simulation Setup

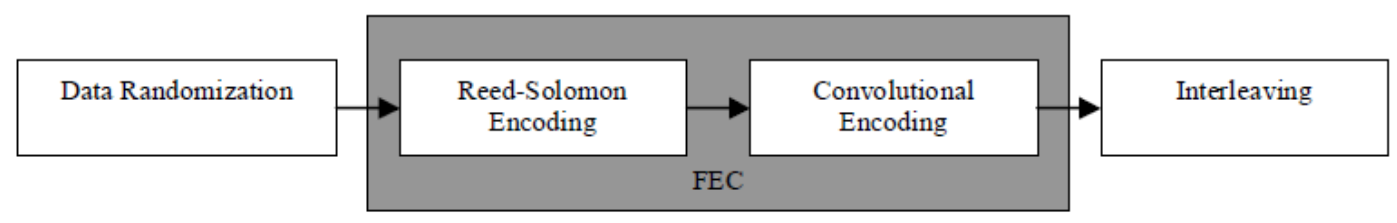

Fig 2: Chaneel encoding setup

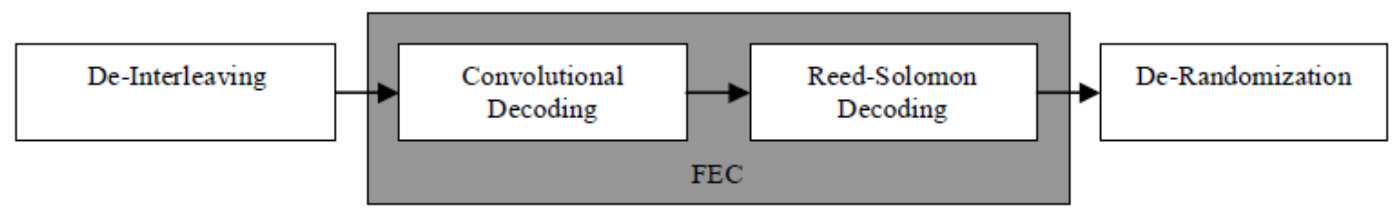

Fig 3: Chaneel decoding setup

\subsection{Scrambler}

The scrambler performs randomization of input data on each burst on each allocation to avoid long sequence of continuous ones and zeros. This is implemented with a Pseudo Random Binary Sequence (PRBS) generator which uses a 15 stage shift register with a generator polynomial of $1+\mathrm{x}^{14}+\mathrm{x}^{15}$ with XOR gates in feedback configuration as shown in figure

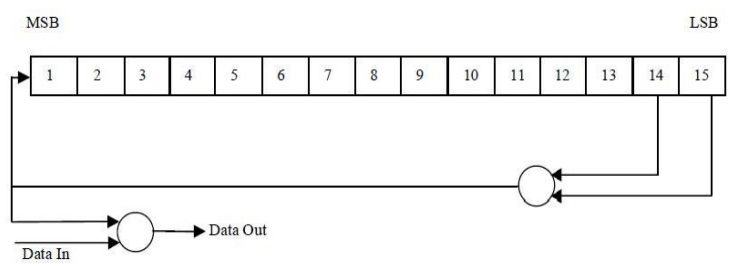

Fig 4: PRBS generator for randomization

\subsection{Reed Solomon coding}

\subsubsection{Encoder}

The randomized data are arranged in block format before passing through the encoder and a single 0X00 tail byte is appended to the end of each burst. The implemented RS encoder is derived from a systematic $\mathrm{RS}(\mathrm{N}=255, \mathrm{~K}=239, \mathrm{~T}=8)$ code using GF $\left(2^{8}\right)$. The following polynomials are used for code generator and field generator:

$$
\begin{gathered}
G(x)=\left(x+\alpha^{0}\right)\left(x+\alpha^{1}\right) \ldots .\left(x+\alpha^{2 T-1}\right), \alpha \\
=02_{H E X} \\
p(x)=x^{8}+x^{4}+x^{3}+x^{2}+1
\end{gathered}
$$


The encoder support shortened and punctured code to facilitate variable block sizes and variable error correction capability. A shortened block of $\mathrm{k}^{\prime}$ bytes is obtained through adding 239k' zero bytes before the data block and after encoding, these 239k' zero bytes are discarded. To obtain the punctured pattern to permit $\mathrm{T}^{\prime}$ bytes to be corrected, the first $2 \mathrm{~T}^{\prime}$ of the 16 parity bytes has been retained.

\subsection{Convolutional Encoder}

The outer RS encoded block is fed to inner binary convolutional encoder. The implemented encoder has native rate of $1 / 2$, a constraint length of 7 and the generator polynomial in Equation (4.3) and (4.4) to produce its two code bits. The generator is shown in Figure 4.5.

$$
\mathrm{G} 1=171 \text { OCT For } \mathrm{X}
$$

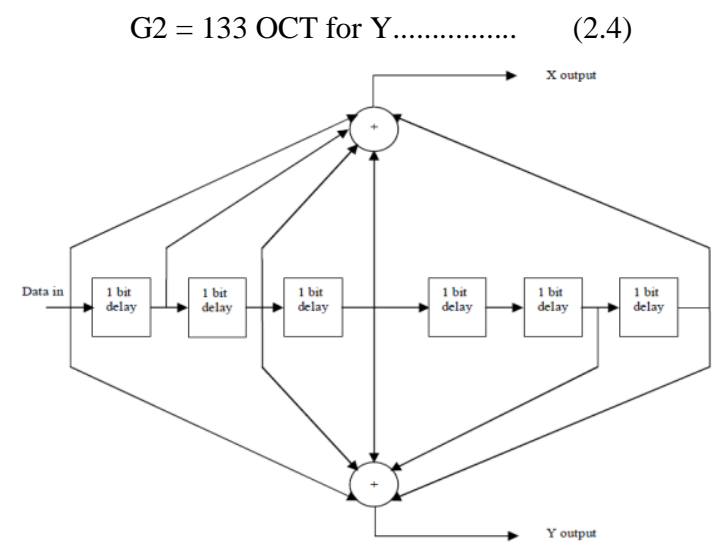

Figure 5.: Convolutional encoder of rate $1 / 2$

In order to achieve variable code rate a puncturing operation is performed on the output of the convolutional encoder in accordance to Table 4.2. In this Table "1" denotes that the corresponding convolutional encoder output is used, while " 0 " denotes that the corresponding output is not used. At the receiver Viterbi decoder is used to decode the convolutional codes.

\subsection{Interleaver}

RSCC encoded data are interleaved by a block interleaver. The size of the block is depended on the numbers of bit encoded per sub channel in one OFDM symbol, Ncbps. In IEEE 802.16, the interleaver is defined by two step permutation. The first ensures that adjacent coded bits are mapped onto nonadjacent subcarriers. The second permutation ensures that adjacent coded bits are mapped alternately onto less or more significant bits of the constellation, thus avoiding long runs of unreliable bits. The Matlab implementation of the interleaver was performed calculating the index value of the bits after first and second permutation using Equation (2.5) and (2.6) respectively.

$$
\begin{array}{r}
f_{k}=\left(\frac{N_{c b p s}}{12}\right) \cdot k_{\bmod 12}+\text { floor }\left(\frac{k}{2}\right) k \\
=0,1,2, \ldots \ldots N_{c b p s} 1
\end{array}
$$

$$
\begin{aligned}
s_{k}=s . \text { floor }\left(\frac{f_{k}}{s}\right)+ & \left(m_{k}+N_{c b p s}\right. \\
& -f l o o r\left(12 \cdot \frac{m_{k}}{N_{c b p s}}\right) \bmod _{(s)} k \\
& =0,1,2, \ldots \ldots N_{c b p s} 1
\end{aligned}
$$

where $\mathrm{s}=$ ceil $\left(\mathrm{N}_{\mathrm{cpc}} / 2\right)$, while $\mathrm{N}_{\mathrm{cpc}}$ stands for the number of coded bits per subcarrier, i.e., 1,2,4 or 6 for BPSK,QPSK 16QAM, or 64QAM, respectively. The default number of sub channels i.e. 16 is used for this implementation. The receiver also performs the reverse operation following the two step permutation using equations respectively.

$$
\begin{aligned}
& f_{j}=s . f \operatorname{floor}\left(\frac{j}{s}\right)+\left(j+f \operatorname{loor}\left(12 \cdot \frac{j}{N_{c b p s}}\right) \bmod _{(s)} j\right. \\
& =0,1, \ldots \ldots N_{c b p s} \\
& s_{j}=12 . . f_{j}-\left(N_{c b p s} 1\right) . \text { floor }\left(12 \cdot \frac{f_{j}}{N_{c b p s}}\right) j \\
& =0,1,2 \ldots \ldots N_{c b p s} 1
\end{aligned}
$$

\subsection{Constellation Mapper}

The bit interleaved data are then entered serially to the constellation mapper. The Matlab implemented constellation mapper support BPSK, grey mapped QPSK, 16QAM, and 64QAMa. The complex constellation points are normalized with the specified multiplying factor for different modulation scheme so that equal average power is achieved for the symbols. The constellation mapped data are assigned to all allocated data subcarriers of the OFDM symbol in order of increasing frequency offset index.

\subsection{IFFT}

The grey mapped data are then sent to IFFT for time domain mapping. Mapping to time domain needs the application of Inverse Fast Fourier Transform (IFFT). In our case we have incorporated the MATLAB 'IFFT' function to do so. This block delivers a vector of 256 elements, where each complex number clement represents one sample of the OFDM symbol.

\subsection{Cyclic Prefix Insertion}

A cyclic prefix is added to the time domain samples to combat the effect of multipath. Four different duration of cyclic prefix is available in the standard. Being $\mathrm{G}$ the ratio of CP time to OFDM symbol time, this ratio can be equal to $1 / 32,1 / 6,1 / 8$ and $1 / 4$

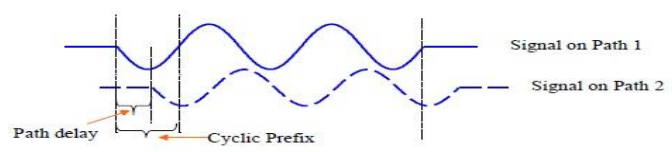

Fig 6 :Cyclic prefix insertion 


\section{SIMULATION RESULTS}

In this chapter the simulation results are shown and discussed. In the following sections, first we will present the structure of the implemented simulator and then we will present the simulation results both in terms of validation of implementation and values for various parameters that characterize the performance of the physical layer.

\subsection{The Simulator}

We have developed the simulator in Matlab ${ }^{\mathrm{TM}}$ using modular approach. Each block of the transmitter, receiver and channel is written in separate ' $\mathrm{m}$ ' file. The main procedure call each of the block in the manner a communication system works. The main procedure also contains initialization parameters, input data and delivers results. The parameters that can be set at the time of initialization are the number of simulated OFDM symbols, CP length, modulation and coding rate, range of SNR values. The input data stream is randomly generated. Output variables are available in Matlab ${ }^{\mathrm{TM}}$ workspace while BER values for different SNR are stored in text files which facilitate to draw plots. Each single block of the transmitter is tested with its counterpart of the receiver side to confirm that each block works perfectly.

\subsection{Physical layer performance results}

The objective behind simulating the physical layer in Matlab ${ }^{\mathrm{TM}}$ was to study BER performance under varying parameters that characterize the performance. But, in order to relay on any results from PHY layer simulation we must have some results that can do some validation in terms of general trends.

\subsection{BER Plots}

In this section we have presented various BER vs. SNR plots for all the mandatory modulation and coding profiles as specified in the standard on same channel models.

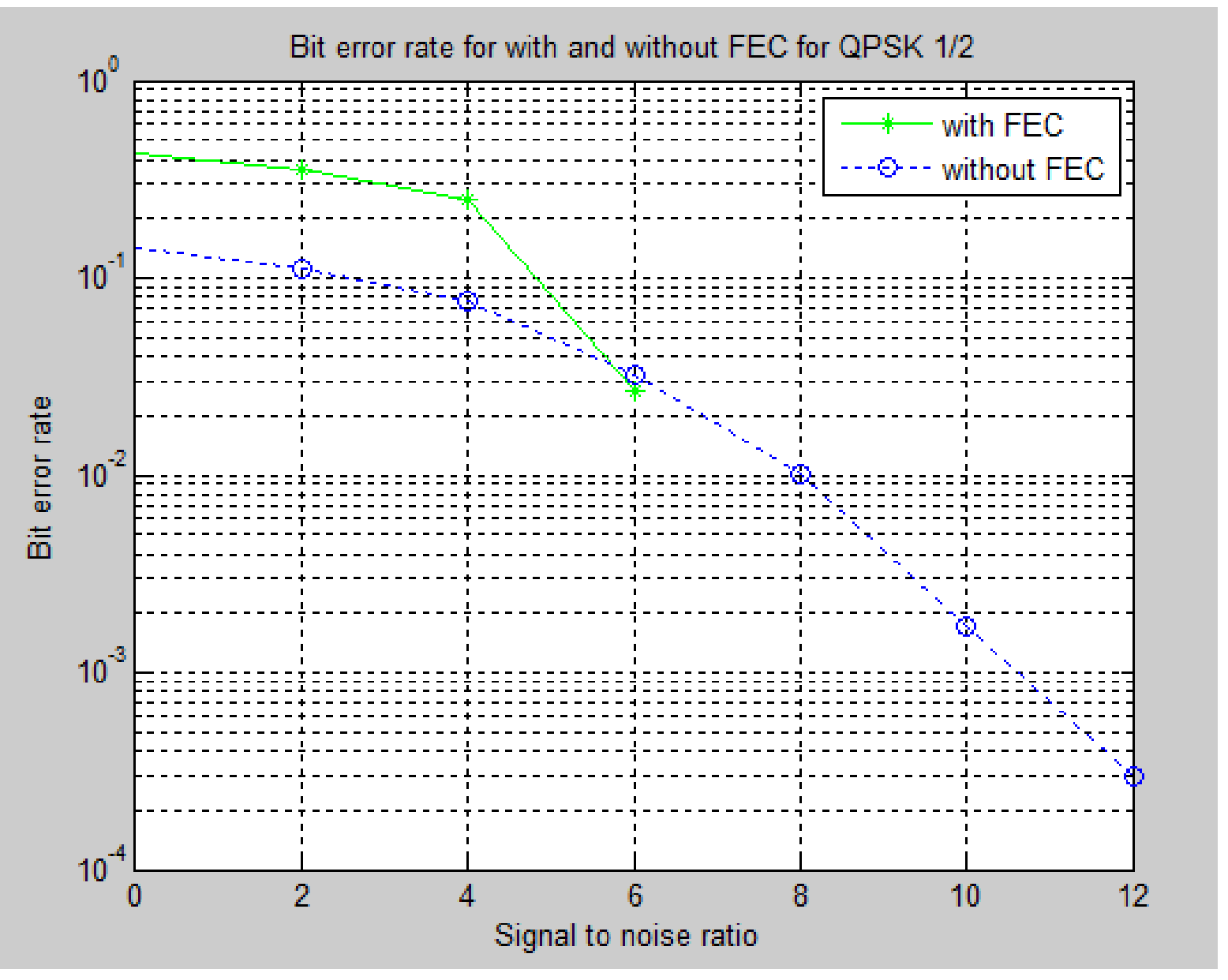

Fig .7 BER vs SNR for QPSK $1 / 2$ with and without FEC 


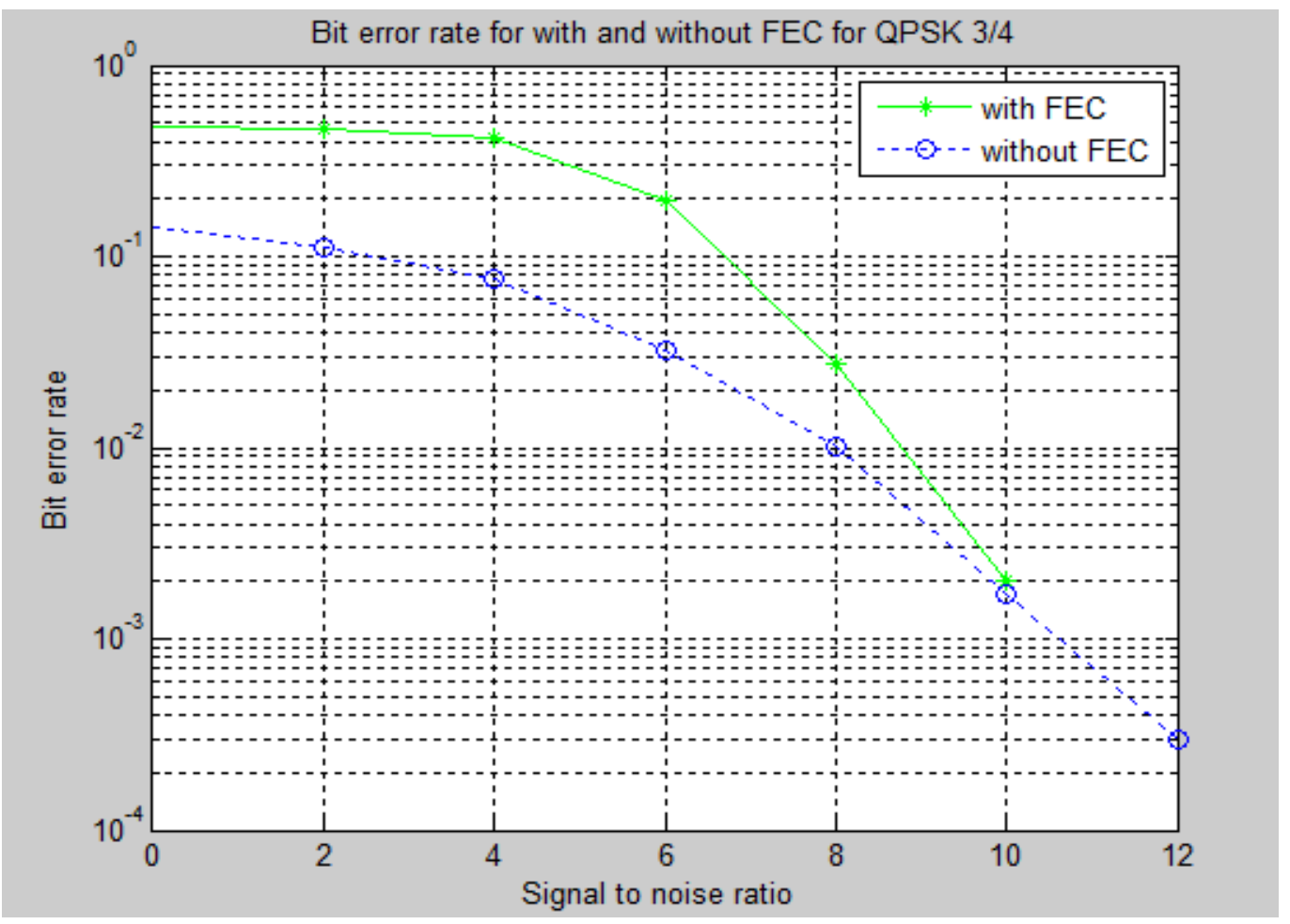

Fig .8 BER vs SNR for QPSK 3/4 with and without FEC

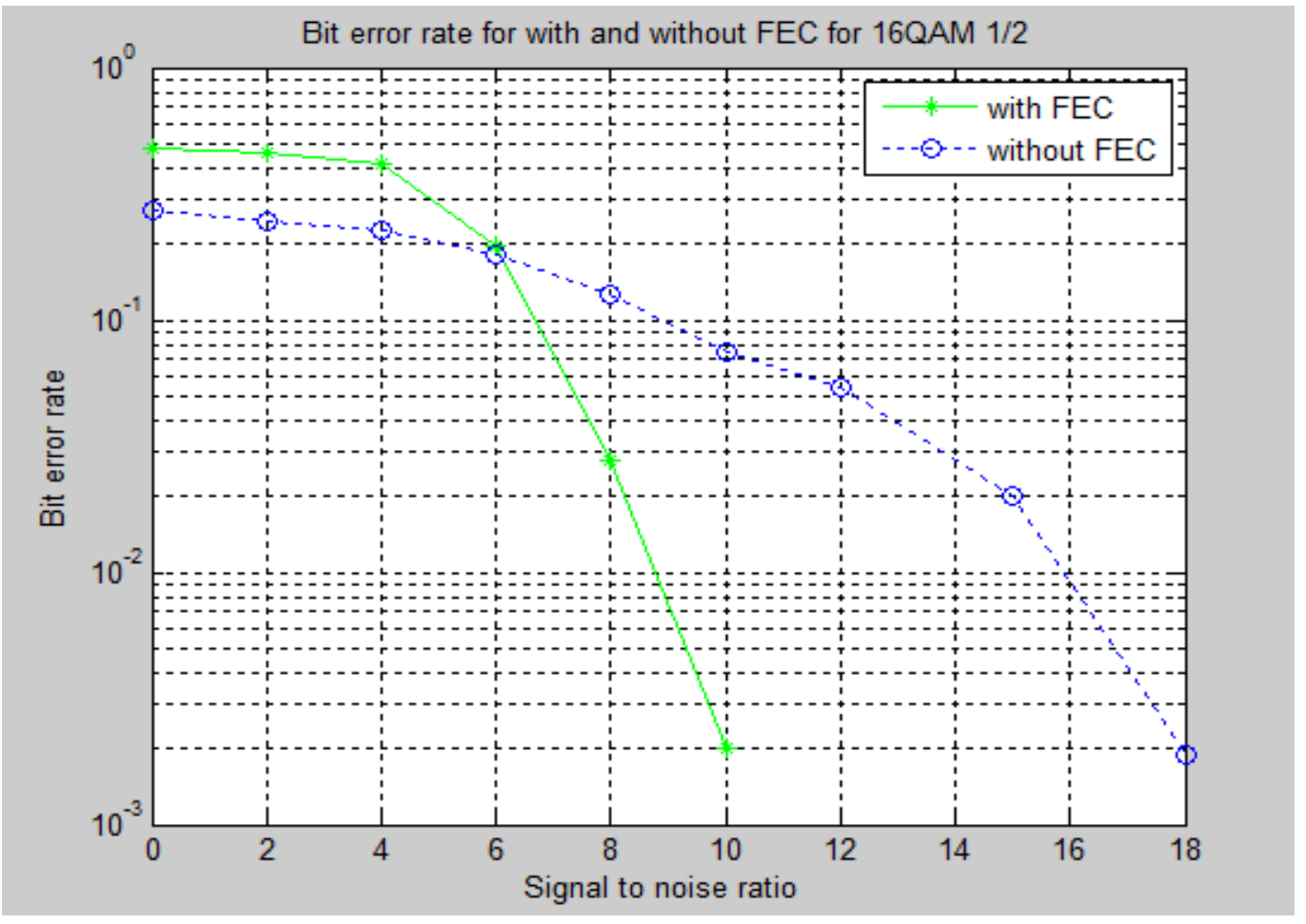

Fig .9 BER vs SNR for 16 QAM 1/2 with and without FEC 


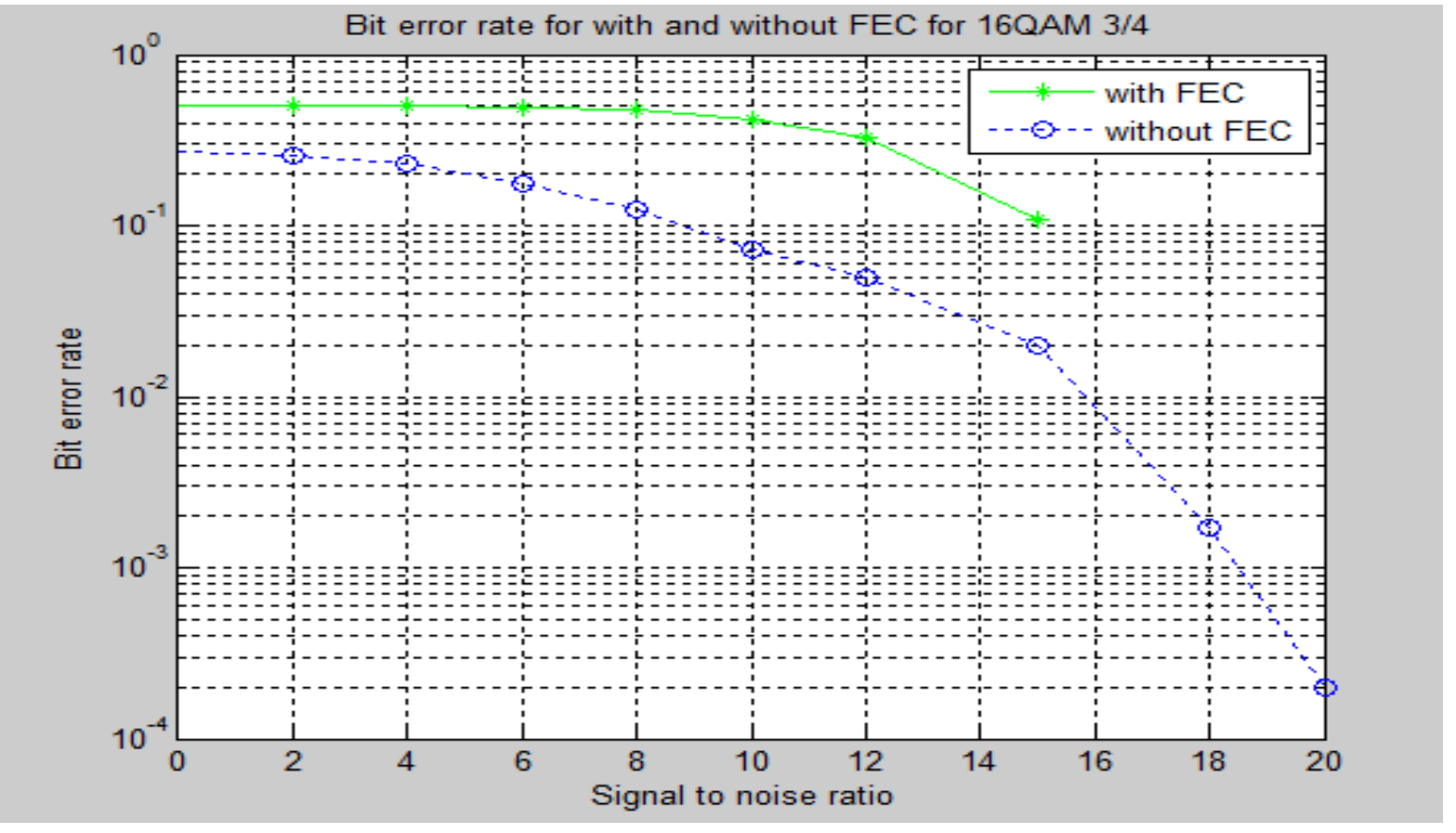

Fig. 10 BER vs SNR for 16 QAM 3/4 with and without FEC

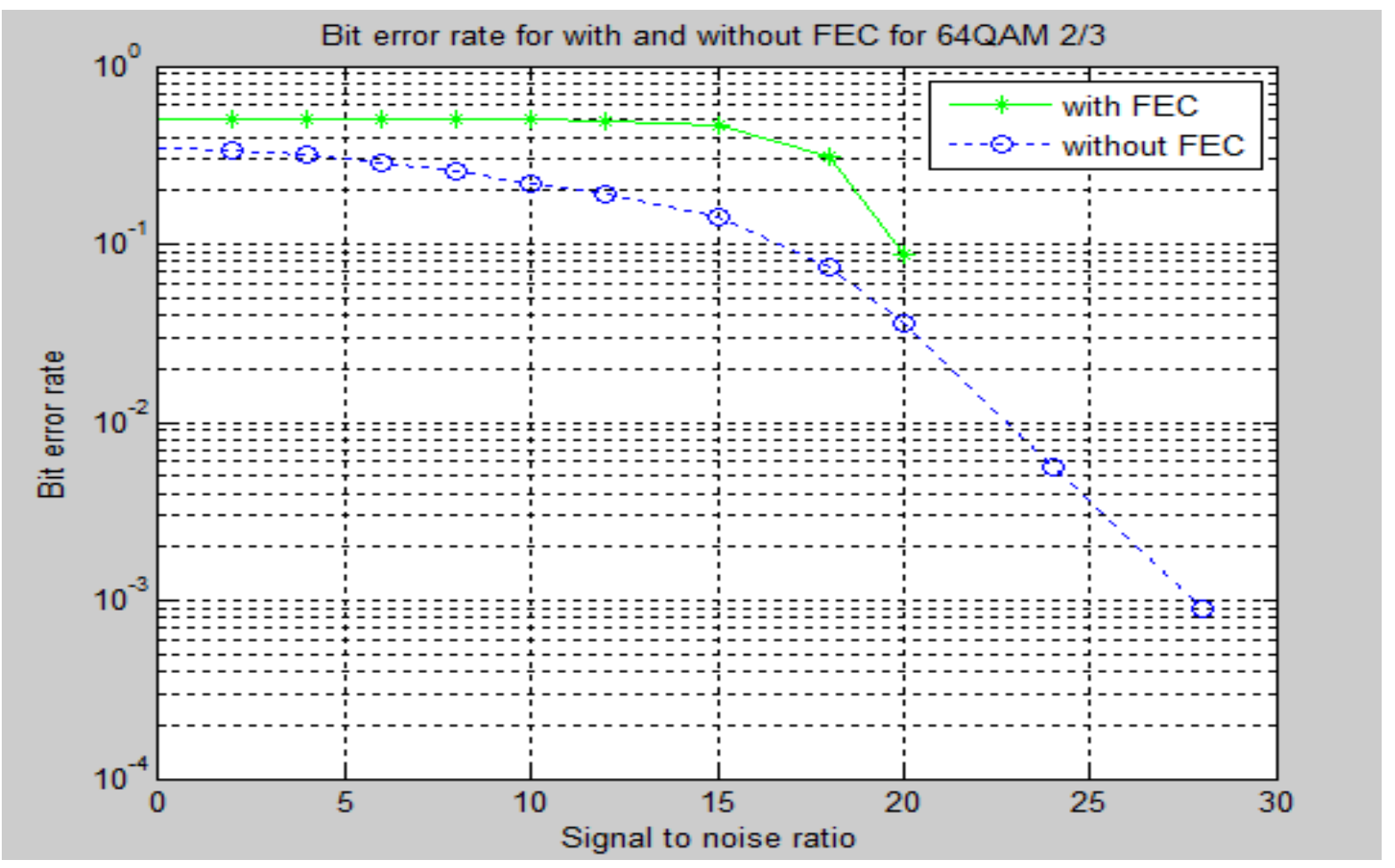

Fig .11 BER vs SNR for 64 QAM $2 / 3$ with and without FEC 


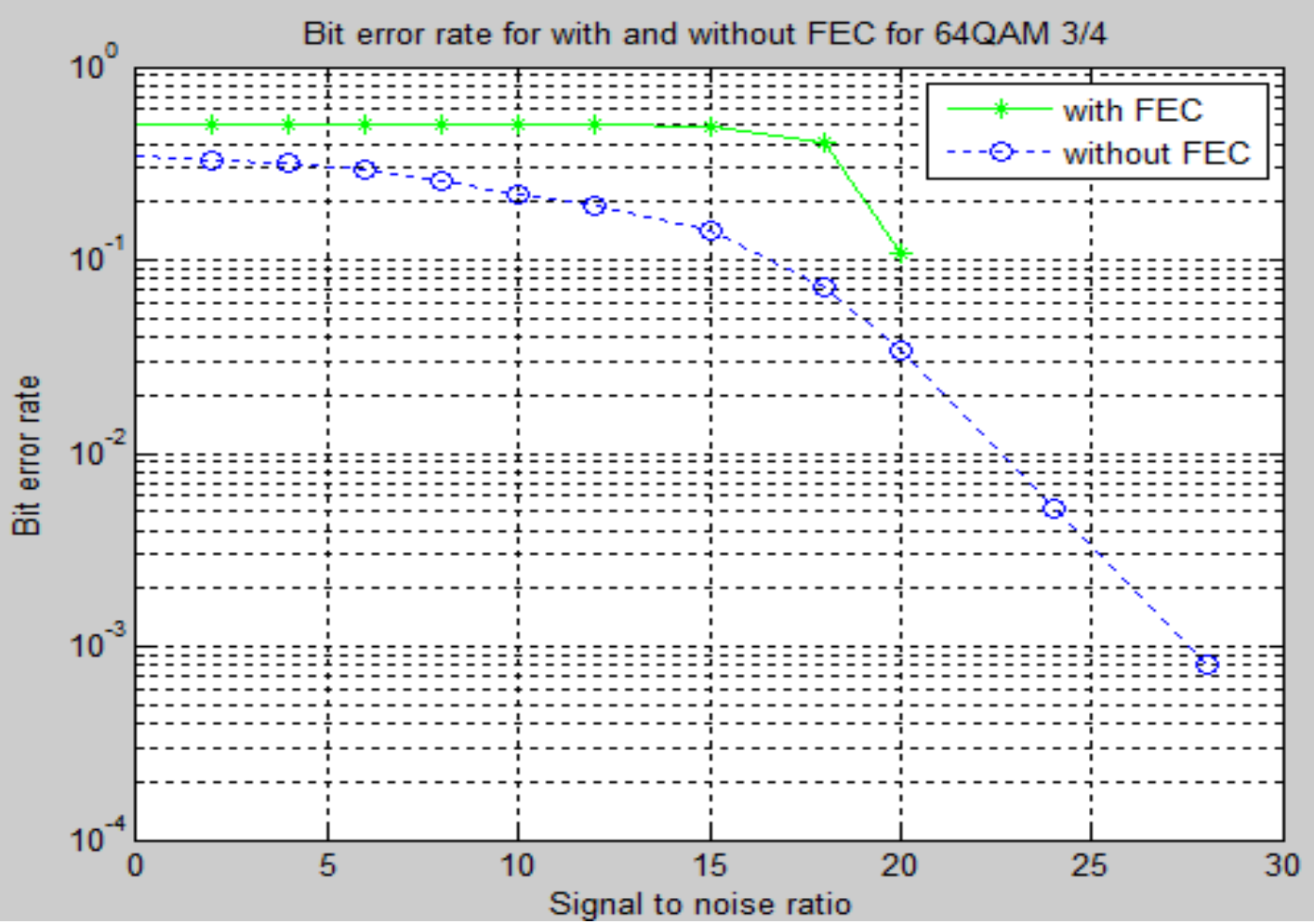

Fig .12 BER vs SNR for 64 QAM 3/4 with and without FEC

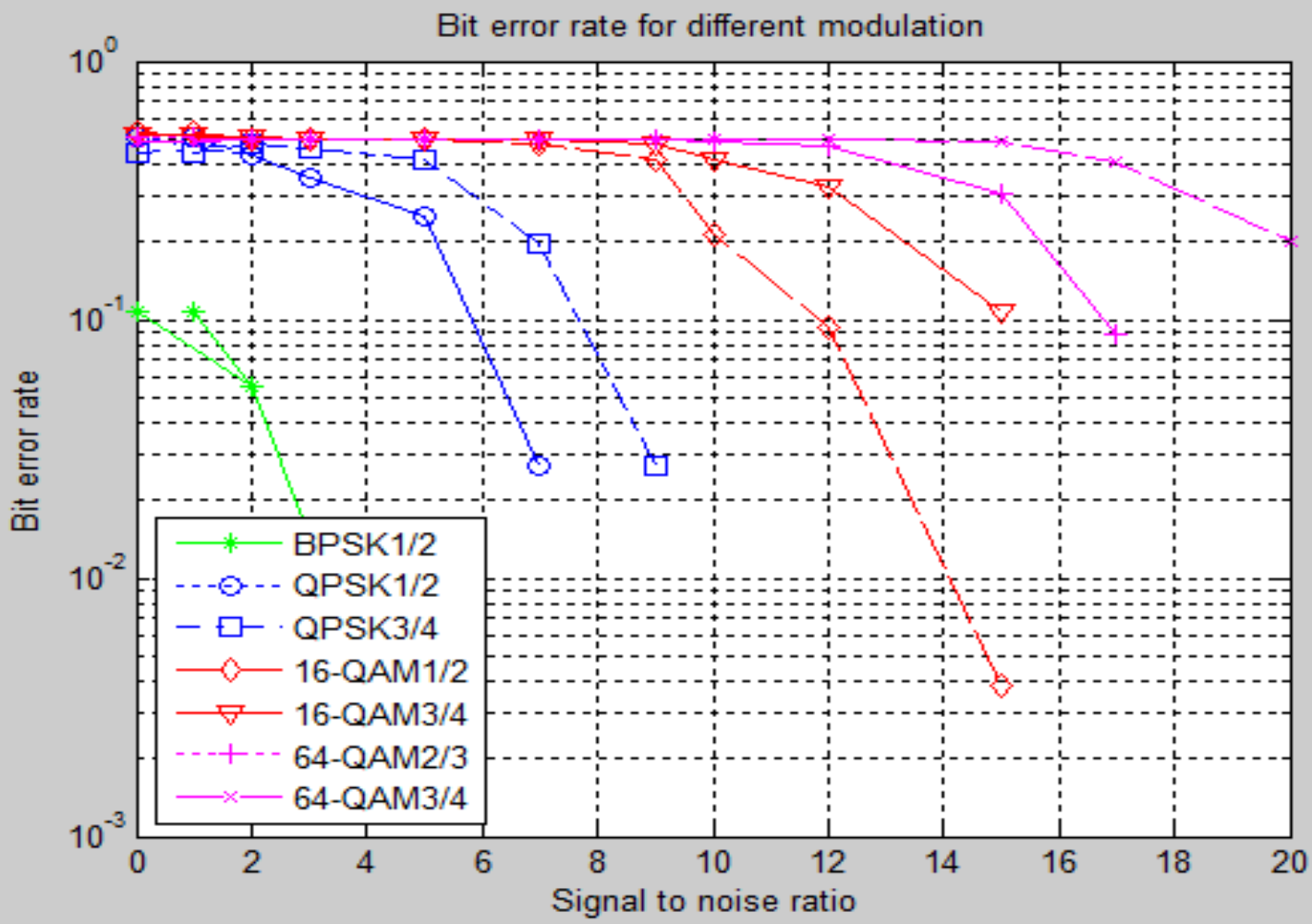

Fig .13 BER VS SNR plot for different modulation techniques 


\section{CONCLUSION}

Error detection and correction techniques are essential for reliable communication over a noisy channel. Reed Solomon codes are one of the most powerful and essential non-binary error correcting codes for detecting and correcting burst errors. The effect of error occur during transmission is reduced by adding redundancy to the data prior to transmission. The redundancy is used to enable decoder in the receiver to detect and correct errors. Cyclic liner block codes are used efficiently for error detection and correction. The encoder splits the incoming data into blocks and processes each block individually by adding redundancy in accordance with a prescribed algorithm and decoder processes each block individually and it correct error by exploiting the redundancy present in the received data. The advantage of cyclic codes in that they easy to encode and process a well defined mathematical structure which has lead to very efficient decoding scheme for them. The reed Solomon codes burst error can be effectively corrected. Reed Solomon codes are efficiently used for compact discs to correct which might occur due to scratches on the discs. Error detection and correction technique are essential for reliable communication over a noisy channel. Reed-Solomon codes are one most powerful and efficient code to correct the errors by using this codes in WIMAX we can reduce the bit-error in noisy environment and useful to provide the efficient data to the subscriber. And by using the OFDM technique, we transmit high data rate in limited bandwidth channel.

\section{REFERENCES}

[1] IEEE 802.162004," IEEE Standard for Local and Metropolitan Area Networks Part 16: Air Interface for Fixed Broadband Wireless Access Systems", 1 October, 2004

[2] Ghosh, A.; Wolter, D.R.; Andrews, J.G.; Chen, R., "Broadband wireless access with WIMAX/802.16: current performance benchmarks and future potential", Communications Magazine, IEEE, Vol.43, Iss.2, Feb. 2005, Pages: 129136

[3] Koffman, I.; Roman, V.," Broadband wireless access solutions based on OFDM access in IEEE 802.16" Communications Magazine, IEEE, Vol.40, Iss.4, April2002,Pages:96103

[4] IEEE 802.16-2006: "IEEE Standard for Local and Metropolitan Area Networks - Part 16: Air Interface for Fixed Broadband Wireless Access Systems".

[5] IEEE Std 802.16-2001,'IEEE Std. 802.162001IEEE Standard for Local and Metropolitan area networks Part 16: Air Interface for Fixed Broadband Wireless Access Systems", December 2001

[6] Derrick D. Boom, "Denial of Service Vulnerabilities in IEEE 802.16 Wireless Networks", Master's Thesis at Naval Postgraduate School Monterey, California, USA, 2004
[7] Hikmet Sari, "Characteristics and Compensation of Multipath Propagation in Broadband Wireless Access System”, ECPS 2005 Conference, 1518 March, 200576

[8] V. Erceg, K.V.S. Hari, M.S. Smith, D.S. Baum et al, "Channel Models for Fixed Wireless Applications", IEEE 802.16.3 Task Group Contributions 2001, Feb. 01

[9] Bernard Sklar, "Digital Communications: Fundamentals and Applications, $2^{\text {nd }}$ Edition,” January 11, 2001

[10] S.Catreux, V.Erceg, D.Gesbert, and R. Heath, "Adaptive Modulation and MIMO Coding for Broadband Wireless Data Networks," IEEE Communications Magazine, pp.108115, June 2002.

[11] WiMAX Forum, "Mobile WiMAX - Part I: A Technical Overview and Performance Evaluation," white paper, August 2006

[12] Y. Xiao, "WiMAX-Mobile Fi: Advanced research and technology, "Auerbach publications, 2008.

[13] L. Nuaymi, "WiMAX: Technology for Broadband Wireless Access,"John Wiley \& Sons, 2007.

[14] S. Ahson and M. Ilyas, "WiMAX: Standards and Security," CRC Press, (Taylor and Francis Group), 2008.

[15] R.B. Marks, K. Stanwood, D. Chang, et al., "IEEE Standard for Local and Metropolitan Area Networks, Part 16: Air Interface for Fixed Broadband Wireless Access Systems," October, 2004

\section{AUTHORS PROFILE}

T. Prabhakar received M.Tech degree from Jawarlal Nehru Technological University Kakinada, Andhra Pradesh, India. B.Tech degree in Electronics and Communication Engineering from SIR C.R.Reddy College of Engineering, Eluru, Andhra Pradesh, India. He is joined as Lecturer in the Department. Of Electronics and Communication Engineering at GMR Institute of Technology, Rajam, Srikakulam District, Andhra Pradesh, India in 2002. Prior to join in this Institute he worked as a Service Engineer in Machine Diagnostics and Deployed to work at National Remote Sensing agency, Department. of. Space, Hyderabad for 1 year 1 month and Trainee Programmer in Indo Tech Computers, for 8 months in Hyderabad. He is presently working as Senior. Assistant Professor in the Department. of Electronics and Communication Engineering at GMR Institute of Technology. Having Total experience is 12 years out of which 10 years in Teaching (GMRIT) and 2 Years in Industry. His research interests are Communication, Signal Processing and Image Processing. He has published 13 technical papers in various International journals and conferences. $\mathrm{He}$ is a life member of ISTE Since 2002. 